Gynecol Obstet Invest 1997;44:283-284

\title{
Subject Index Vol. 44, 1997
}

Gynecologic and Obstetric Investigation

Adenomyosis 200 Adhesion formation 115 Amniocentesis 217 Amnioinfusion, transabdominal 249 Amniotic fluid index 234, 249 Ampicillin/sulbactam 21 Amylin 6 Analgesics 255

Androgens 127 Angiogenesis 107 Anxiety 157

B-hemoglobin 163 Bishop score 224 Blood loss 163 Body mass index 127 Bone density 127

Brain malformations 206

- $\quad$ metastasis 214

Breech presentation 169

c-fos 93

ojun 93

c-myc 136

Cancer 270

Candida albicans 16

Carboplatin 196

Cardiotocography 234

Catecholamine 173

Cefotetan 21

Cervical cancer 57, 136,278

- polyps 191

Cesarean section 169,173,234 Chemotherapy 70 Choriocarcinoma 211 Cisplatin 57,270

Computer cardiotocography 249 Continuous epidural analgesia 73

- $\quad$ local injection 196

Cornual pregnancy 64

Counselling 182

Cytogenetic analysis 217

Delivery 163

Dermatology 260

Diabetes 11

Direct intraperitoneal insemination

149 Distribution 270 Doppler echocardiography 173 Dyspareunia 53

Echogenicity assessment 244 Embolization 211 Embryo transfer 157 Endometrial implant 32

- polyps 191

Endometriosis 38

Endometritis 21

Endometrium 32, 187

Endothelial cells 47

- $\quad$ constitutive nitric oxide synthase

221

Endothelin-1 93,221 Epidural metastasis 214 17ß-Estradiol 47 ET-A receptor 221 Ethanol 153 
Fallot's tetralogy 61 Feminine hygiene 260 Fertility rate 239 Fetal distress 234

fibronectin 224

heart rate pattern 26

- -, short-term variability 249

hypoxia 229

liver, computed sonography 244

malformations 107

- $\quad$ monitoring 26

Follicular fluid 145

Free oxygen radicals 229

Gestational diabetes 177

- $\quad$ - mellitus 6

Gianturco coils 211

Glucose transporters 89

Grief process 157

Gynecology 260

H-score 132

Heteroduplex 136

Histiocytosis X 67

Histology 115

Hormone replacement therapy 47, 124,

187 Human endometrial cancer 132

milk 102

papillomavirus 53

placenta 153 Hydatidiform mole 221 Hydranencephaly 206 Hydrogen-peroxide-producing lactobaciUi

16

Hypertensive disorders 11 Hypospadia 107 Hysteroscopy 191

Image density 244 Immunohistochemistry 32, 132 In vitro fertilization 157 Infertility 157

Insemination 265 Insulin-like growth factor 145

- factor-1 127

- factor-binding protein 145 Interferon 53

Intra-arterial infusion 57 Intracytoplasmic sperm injection 1 Intraperitoneal insemination 239

Intrauterine growth retardation 89, 93

- $\quad$ insemination 149

Islet amyloid polypeptide 6

Jordan 169

Labor 73

- $\quad$ stress 173

Langerhans cell histiocytosis 67 Laparoscopy 38 Latency period 249 Leiomyoma 275

Leiomyosarcoma 70 Ley dig cell tumour 141 Lipid peroxidation 229 Local anesthetics 73

Lumbar epidural analgesia 73 Lymph node 270

Macacafascicularis, mammary gland 41

Macrosomia 89

Magnesium sulfate 82

Malignant mixed müllerian tumor 214 
Maternal diabetes mellitus 89

Medical abortion 112

Menopause 187, 191

Methotrexate 64, 112

Microbiology 260

Minimal endometriosis 32

Minimally oxidized low-density lipoprotein

47 Misoprostol 112 Missed abortion 217 Monocyte adhesion 47 mRNA 221

- $\quad$ expression 93

KÀRGER

E-Mail karger@karger.ch Fax+ 41613061234

http:// www. karger. ch

(C) 1997 S. KargerAG, Basel

283

Multifetal pregnancy reduction 1 Multiple pregnancy 1 Mutation 136

Nd:YAG laser 38, 115 Neonatal bone abnormality 82 Nitric oxide 153

- $\quad$ - synthase 177

Nonepithelial ovarian tumour 141

Obesity 120

Oligohydramnios sequence 206 Oocyte maturation 145 Opioid narcotics 73 Oral glucose

tolerance test 6 Ovarian carcinoma 278

superovulation 149

tumour 141 Ovary 275

Ovulation induction 115 Oxygen administration 26

Pain relief 255

Parathyroid-hormone-related protein 102 Patient-controlled epidural analgesia 73 Perineoplasty 53 Placenta 6,93,221 Placental vasculopathy 107 Polycystic dysplastic kidneys 206

- $\quad$ ovarian syndrome 120

Polymorphonuclear leukocytes 16

Postmenopausal women, prolactin 124

Postmenopause 200

Postterm pregnancy 224

Potter syndrome 206

Preeclampsia 82, 93, 97, 107

Pregnancy 11

-, complication 206

-, postoperative infections 21

Premature ovarian failure 127

rupture of membranes, preterm 249 Prenatal diagnosis, Fallot's tetralogy 61 Preterm breech 169

delivery 249 Primary dysmenorrhea 255 Progesterone 47 Prognostic factors 239 Prolactin 120

-, postmenopausal 124 Proliferating cells 41 Prolonged pregnancy 16 Psychometrics 157

Puerperium 163 Pulmonary hypertension 173

Random allocation 149 Randomized controlled trial 21 ras p21 protein 132 Refertilization 182

Reflectance pulse oximetry 26 Reflexes 265

Sanitary pad 260 
Semen 265

Spinal cord compression 214

Squamous cell carcinoma 278

Sterilization 182

Steroid receptors 41

Stress 157

Surgical wound infection, prophylaxis 21

Tcell 32

Tamoxifen 200

Term pregnancy 234

Tissue platinum concentration 196

Tocolysis 82

Transcutaneous electrical nerve stimulation

255 Transvaginal puncture 64

- $\quad$ sonography 115

Twin pregnancy 97

Ultrasound 187,234,244 -, hydrops fetalis 61 Umbilical cord blood 229 Uric acid 97 Uterine cancer 196 Uterus 265, 275

Vagina 265 Vaginal bleeding 211

delivery 73, 169, 173

wall 275 Vasoconstriction 153 Vestibulitis 53 Villous vascular tree 177 Vulva 67

Vulval irritation 260

284

Gynecol Obstet Invest Vol. 44, 1997

Subject Index 